\title{
COMPETENCIA INTERCULTURAL EN EL ÁMBITO SANITARIOS: EL CASO DEL NACIMIENTO TAILANDÉS
}

\author{
Maneerat Marnpae \\ University of Alcalá, Spain \\ maneerat.marnpae@gmail.com
}

\begin{abstract}
Resumen: Durante los últimos años la afluencia migratoria está generando nuevas necesidades en la mayoría de los países, y un ejemplo de este fenómeno es el afloramiento de necesidades comunicativas en diferentes ámbitos siendo uno de ellos el ámbito sanitario. A raíz de esto se han aumentado las diferencias lingüísticas y culturales en la sociedad receptora. Para lograr una atención sanitaria eficaz, sería imprescindible que personas involucradas con dicho trabajo (personal sanitario, traductor, intérprete, mediador, trabajador social, etc.) conocieran la cultura y tradiciones de los usuarios en cuestión, ya que aquellas forman parte de sus hábitos y conductas relacionados con la salud. Este artículo tiene como objetivo fomentar la competencia intercultural y calidad de formación de las personas anteriormente mencionadas. En las páginas que siguen se presentarán aspectos culturales relativos a las creencias, costumbres y cultura material tailandeses en el ámbito de la salud y el caso del nacimiento, que podrían resultar útiles para la atención sanitaria/traducción e interpretación, etc. El estudio nos lleva a la conclusión de la necesidad de incorporar conocimientos culturales en el ámbito sanitario.
\end{abstract}

Palabras clave: Competencia intercultural, cultura sanitaria, traducción-interpretación, el caso del nacimiento, usuario tailandés.

\begin{abstract}
In recent years, the migration flow has created new needs in most countries, for instance, the emergence of communication needs in different settings, such as in a field of public health. As a result, linguistic and cultural differences in the host society have been increased. In order to achieve an effective medical care, it would be essential that people involved with such work (health personnel, translator, interpreter, mediator, social worker, etc.) knew the users' culture and traditions, since those are part of their health-related habits and behaviors. This article aims to promote the intercultural competence and quality of training of the persons mentioned above. In the following pages the cultural aspects related to Thai beliefs, customs and material culture in the healthcare setting and the birth case will be presented, that could be useful for medical care/translation and interpretation, etc. The study leads us to the conclusion that we need to include cultural knowledge in the healthcare setting.
\end{abstract}

Key words: Intercultural competence, health culture, translation-interpretation, the birth case, Thai user.

\section{Introducción: Importancia de la comunicación correcta en la atención de la salud entre el personal sanitario autóctono y el usuario extranjero}

La sociedad en la que vivimos hoy en día cada vez existe más la tendencia de las sociedades multiculturales, y entre los países occidentales se encuentra España, que se ha convertido en una sociedad multilingüe y multicultural, debido a que los miembros de otros países traen consigo sus culturas y/o lenguas diversas a la sociedad receptora. Los nuevos habitantes también utilizan los servicios públicos (SP) tan importantes, al igual que los autóctonos, como, por ejemplo, la sanidad, la enseñanza, la vivienda, los servicios sociales y procedimiento jurídico. Su llegada ha hecho que surjan una serie de necesidades nuevas en el panorama de los SP, puesto que en la mayoría de las ocasiones los nuevos habitantes limitan la comunicación interlingüística y se requieren soluciones. Las barreras lingüísticas y culturales son cada vez más patentes, por tanto, los proveedores de los SP han tenido que 
buscar soluciones para atender a aquellos que no hablaban español o con los que las diferencias culturales impedían una correcta comunicación, con la finalidad de mejorar la comunicación entre los usuarios extranjeros con los profesionales del país.

Flores (2000; citado en Angelelli 2004: 19) afirma que sólo con el hecho de las discrepancias culturales (sin contar con la presencia de las diferencias lingüísticas), éstas pueden obstaculizar una comunicación eficaz y conllevar a los malentendidos. Una comunicación ineficaz puede generar múltiples inconvenientes sobre la atención de la salud, como, por ejemplo, un diagnóstico equivocado, procedimientos arriesgados, tratamientos innecesarios, sufrimientos innecesarios (Haffner 1992; citado en Samovar et al. 2007: 285) e incluso la ineficacia de los tratamientos, como el caso de los usuarios chinos y surasiáticos que suelen responder de forma afirmativa a la pregunta de los médicos españoles acerca de si han seguido el tratamiento, aunque en realidad no lo han hecho. Esto se debe a que dichos pacientes no suelen confrontar directamente con el otro, especialmente si el otro se considera jerárquicamente superior (Raga Gimeno y Sales Salvador 2010).

Para prevenir los problemas anteriormente indicados, los profesionales sanitarios deben ser culturalmente sensibles y conscientes de que su forma comunicativa es culturalmente congruente con la información de tratamiento y salud (Purnell y Paulanka 1998; citado en Samovar et al. 2007: 285). Dicho de otro modo, deben poseer una competencia intercultural al ponerse en contacto con los usuarios extranjeros. La comunicación intercultural en el ámbito de la salud no sólo se manifiesta en encuentros interculturales entre el personal sanitario y el usuario extranjero, sino también incluyen terceras personas, como por ejemplo, el intérprete, mediador, trabajador social, etc. También estas personas indicadas requieren una competencia intercultural a la hora de prestar sus servicios.

\section{Competencia intercultural}

Por regla general, la competencia intercultural se refiere a las habilidades eficaces en la interacción entre culturas; y los contextos en los que se desarrolla la interacción intercultural son diversos como, por ejemplo, en una interacción de la vida cotidiana, negocios, educación, militares, etc. Wiseman (2002: 208) define la competencia comunicativa intercultural como: "ICC competence involves the knowledge, motivation, and skills to interact effectively and appropriately with members of different cultures". Mientras, Chen y Starosta (2005: 241) son más específicos con respecto a la definición de la competencia intercultural, pues la definen como "the ability to effectively and appropriately execute communication behaviors to elicit a desired response in a specific environment"; es decir, estos dos autores destacan la importancia de un entorno específico en su definición. Con el fin de interactuar eficaz y adecuadamente es imprescindible que un individuo posea suficientes conocimientos culturales y motivaciones o actitudes a fin de aplicarlos en las destrezas comunicativas.

Varios autores identifican los tres componentes principales del desarrollo de la competencia intercultural que debe tener un comunicador competente como los componentes más comunes, siguiendo la propuesta de Spitzberg y Cupach (1984): la motivación/actitudes (el proceso emotivo), los conocimientos (el proceso cognitivo) y las destrezas (el proceso conductual) (Spitzberg y Cupach 1984, Chen y Starosta 1996, Hofstede 1997, Gudykunst 1998, Ting-Toomey 1999, Spencer-Oatey y Franklin 2009, Lustig y Koester 2010). La finalidad de los componentes enunciados sería permitir al comunicador que tenga éxito en la interacción intercultural de forma (más) eficaz y también (más) apropiada. Los componentes pueden modificar la interacción, establecer la competencia o incluso aumentarla. En este artículo enfatizamos sólo el segundo componente de la competencia intercultural, los 
conocimientos o el proceso cognitivo, ya que en los próximos apartados hablaremos de información de aspectos culturales que son útiles en la atención de la salud.

Según Triandis (1977), el proceso cognitivo hace hincapié en el cambio del pensamiento personal sobre el entorno a través de la comprensión de diferentes características de sus propias culturas y las de los demás. Esto es, se refiere al entendimiento de las convenciones culturales que afectan a los pensamientos y comportamientos de los individuos. Chen y Starosta (1996: 366) definen el proceso cognitivo como "[...] individuals tend to be more competent in intercultural communication as they acquire greater degrees of cultural awareness and self-awareness". Es decir, primero, uno tiene que poseer el conocimiento de su propia identidad siendo consciente de las propias características culturales y de sus procesos comunicativos. Segundo, se tiene que tomar conciencia del proceso de comunicación intercultural, y para comprender a los demás uno debe comprenderse primero a sí mismo.

Lustig y Koester (2010: 70) parten de la distinción del conocimiento o la información cognitiva entre dos tipos: 1) la información general sobre la cultura, como es por ejemplo, el conocimiento sobre la comunicación interpersonal y la influencia cultural en el proceso comunicativo; y 2) la información específica sobre la cultura con el objetivo de llegar a comprender las culturas particulares, que puede ser la información de costumbres específicas en la comunicación interpersonal de aquella cultura. Sin embargo, Spencer-Oatey y Franklin (2009: 202) afirman que los conocimientos consisten en varios aspectos, por ejemplo, el conocimiento referente a los valores y normas de culturas particulares que tenemos en contacto con las personas de aquellas culturas, conocimiento contrastivo de culturas en general, o el conocimiento del proceso de interacción intercultural; y además, para lograr el éxito en una interacción intercultural, los conocimientos requieren el uso de destrezas, modificaciones y adaptaciones de actitudes, perspectivas y valores necesarios.

De esta manera, el hecho de poseer el conocimiento se considera muy importante, puesto que un comunicador carente del conocimiento o la información necesaria para un encuentro intercultural podría cometer errores en interpretar el mensaje recibido, seleccionar incorrectamente las estrategias comunicativas, violar las normas o reglas de la cultura y la comunicación en una situación dada, o incluso no ser capaz de atribuir los motivos de error o fallo que ha cometido y tampoco poder resolver un problema comunicativo determinado. Todo lo expuesto anteriormente podría suceder en la atención sanitaria a usuarios extranjeros si las personas involucradas (personal sanitario, traductor-intérprete, mediador, trabajador social) carecen de los conocimientos necesarios, como son por ejemplo, aspectos culturales en la sanidad del país de origen, cultura material y cultura comunicativa de los usuarios.

\section{Creencias, Costumbres y Cultura Material tailandeses en el ámbito de la salud}

Las diferencias culturales pueden generar problemas comunicativos en encuentros interculturales entre personas de origen diverso. Por lo que respecta a los estudios de comunicación intercultural, el Grupo CRIT (2006) parte de la distinción del término cultura, que es muy difícil de definir, en dos tipos: la cultura material y la cultura comunicativa. La primera se refiere a las costumbres en general y los aspectos relacionados con la vestimenta, la gastronomía, el hogar, el trabajo, las fiestas, entre otros. No compartir la información básica de las personas de diferentes culturas puede causar problemas comunicativos. Con respecto a la cultura comunicativa, hace referencia a la forma de conversar y de interactuar, sus discrepancias resultan más graves que en la cultura material debido a que presentan un grado mucho mayor de inconsciencia o invisibilidad.

En este apartado nos centraremos en las Creencias, Costumbres y Cultura Material, cuyos detalles se darán en páginas sucesivas que contienen todo aquello que no es cultura 
comunicativa. Es decir, todos aquellos comportamientos que no intervienen directa y específicamente en las interacciones comunicativas. Nos limitaremos sólo a tratar la cultura material ya que consideramos que el hecho de comprender los sistemas de creencias de un paciente se considera un primer paso vital hacia las prestaciones sanitarias culturalmente competentes, lo cual podría generar una comunicación óptima y mejorar los efectos sobre la satisfacción de los pacientes de la atención prestada.

A continuación hablaremos de concepciones culturales del cuerpo humano, la salud y la enfermedad de la sanidad tailandesa antes de centrarnos en detalle con el fenómeno del nacimiento tailandés.

\subsection{Concepciones culturales del cuerpo humano, la salud y la enfermedad}

En la sanidad de Tailandia se practican la biomedicina, la medicina tradicional (de carácter holista y ayurvédico) y las prácticas mágicas-religiosas. Se cree que la curación a través de muchas maneras diferentes les ayudará a mejorar la eficacia, por tanto, algunas personas mezclan el uso de la medicina moderna con otras formas de tratamiento como complemento o alternativa: la medicina tradicional tailandesa, las hierbas tailandesas y chinas, la práctica de magia (por ejemplo, el uso de amuletos de los monjes budistas que se cree que pueden proteger a los portadores de la mala suerte o de peligros y enfermedades) (Salguero 2003, 2006, 2007, Waikagul 2008, Marnpae 2011).

Entre las características de la medicina tradicional de Tailandia, podemos destacar su carácter holista y ayurvédico. El carácter holista es un tratamiento del conjunto del organismo en el aspecto físico y psicológico. Ayurveda es un sistema de la medicina alternativa que trata e integra cuerpo, mente y espíritu. Este emplea un enfoque holista comprensible, poniendo especial énfasis en el uso de una dieta, tratamiento herbal, ejercicio, meditación, terapia física, etc. El concepto del equilibrio es fundamental, porque cuando suceda el desequilibrio corporal, esto afectará al funcionamiento de todo el cuerpo.

Según la medicina tradicional tailandesa, el círculo de la vida consta de tres elementos importantes: cuerpo, mente-corazón y energía. Los tres elementos indicados funcionan juntos y tienen relación entre sí. Si uno de esos tres pierde el equilibrio, puede causar el mal funcionamiento de la salud y de la inmunidad, dejando el organismo vulnerable a cualquier enfermedad. Consecuentemente, a la hora de sanar a un enfermo deberán tratarse varios problemas simultáneamente tanto en el aspecto físico, mental como enérgico, con la misma atención de cada esencia (Salguero 2006).

Además, el cuerpo humano está formado por cuatro elementos: Tierra, Agua, Aire y Fuego. Cada elemento no hace referencia a la sustancia física, sino a las cualidades de la sustancia. Las sustancias sólidas pertenecen al elemento Tierra. Las sustancias líquidas se engloban dentro del elemento Agua. El movimiento es la cualidad del elemento Aire. El calor es la cualidad del elemento Fuego. Cuando los cuatro elementos en nuestro cuerpo están en equilibrio, tendremos una salud buena y saludable. De lo contrario, si ocurre un desequilibrio con estos elementos, podríamos sufrir ciertos síntomas o enfermedades (Salguero 2003, 2007, Chokevivat y Chuthaputti 2005).

Se cree que las causas de enfermedades podrían provenir del poder supernatural, de la naturaleza, del universo, aparte del motivo según la diagnosis de la biomedicina. Por eso, el médico de la medicina tradicional tendrá que realizar diversos tipos de reconocimientos: el reconocimiento físico, astrológico y la diagnosis. Aparte del uso del método de la biomedicina para curarse, al mismo tiempo, en ciertos casos algunos buscan otros métodos alternativos como, por ejemplo, la medicina tradicional tailandesa, acudir a un monje budista, un chamán o un astrólogo. Los dos penúltimos casos son llevados a cabo para realizar los rituales, y el último caso es utilizado con el fin de determinar sus destinos y buscar una 
solución. En algunos casos se considera que la enfermedad mental está producida por causas sobrenaturales, hechizos o maldiciones. Se consulta con un monje budista o chamán para que realice una prognosis del problema y una ceremonia para sanar al paciente (Chokevivat y Chuthaputti 2005, Salguero 2006).

\section{El caso del nacimiento}

En este apartado se tratarán las prácticas relativas al fenómeno del nacimiento que engloban creencias, costumbres realizadas para las nuevas madres y los recién nacidos, así como los métodos alternativos para cuidar la salud de las parturientas.

\subsection{Costumbres del nacimiento}

En el pasado el embarazo, parto y nacimiento eran peligrosos a causa de la falta de desarrollo en la medicina y las ciencias. Por consiguiente, se desarrollaron ciertos principios basados en creencias, costumbres y ceremonias sobre el nacimiento, los cuales se practicaban esencialmente por las siguientes razones (Sathiankoset 1996): 1) evitar el peligro en las embarazadas; 2) facilitar el parto y evitar cualquier peligro, incluso el fallecimiento de mujeres durante el parto, hay un dicho tailandés para las embarazadas que viene a decir que el parto es como una guerra para las mujeres, porque no se sabe si sobrevivirán tras el parto; $\mathrm{y}$, por último, 3) proteger al recién nacido, pues se considera que es muy sensible y susceptible al fallecimiento durante los primeros meses de su vida. Además, existen creencias y ceremonias según las cuales el niño crecerá con una buena salud.

Tradicionalmente, estas tres razones están relacionadas con la creencia basada en evitar y expulsar a los espíritus que pueden perjudicar la vida de madres y bebés. Cuando algo malo sucedía durante el embarazo, parto o posparto, la gente pensaba que había sido obra de los espíritus.

Para ponerle nombre a un niño, como los tailandeses son muy apasionados por la astrología, consultan a un astrólogo o un libro que trate sobre el significado de los nombres con el fin de tener un nombre auspicioso para el recién nacido (Salguero 2006). Hay que considerar el día y la hora de su nacimiento, así como su sexo para los cálculos astrológicos. Por regla general, los tailandeses cuentan con dos nombres: un nombre oficial, el cual se inscribe en el registro y otro nombre familiar o nick name, que es más corto que el nombre oficial. El nombre familiar se utiliza con más frecuencia que el nombre oficial, pues resulta más fácil pronunciarlo al ser más breve, y suelen utilizarse entre familiares, amigos, colegas o en ciertas ocasiones que no son formales.

En los momentos posteriores al parto, algo que puede llamar la atención y generar cierto malentendido es la prohibición de alabar al neonato. Esto tiene como objetivo evitar el secuestro del recién nacido por parte de los espíritus y cualquier consecuencia mala que pudiera suceder por culpa de obras llevadas a cabo por los espíritus como por ejemplo, la enfermedad y la muerte. Tradicionalmente, si sus hijos siempre están enfermos, sus padres piden a un monje budista que aten hilos sagrados en sus muñecas para evitar a los espíritus. Se cree que cuando un niño está frecuentemente enfermo es debido a la obra de los espíritus. Si sus niños están bajo el cuidado de un monje budista, se cree que ellos están seguros ya que los espíritus temen a los monjes (Sathiankoset 1996).

Después del alumbramiento, por regla general, se celebran rituales y ceremonias relativos con el alma o espíritu protector en cada persona (ขวัญ $/ \mathrm{k}^{\mathrm{h}} \mathrm{wu}: \mathrm{an} /$ ) para un bebé, con el fin de darle la bienvenida y mantener el espíritu protector. Éste se considera la esencia de la vida, su pérdida o abandono podría generar diversos efectos como por ejemplo, una 
enfermedad e incluso el caso más crítico que sería el fallecimiento del niño. Esto se debe a que los niños son los más susceptibles a perder el espíritu protector por el susto o shock.

En la relación con el calostro, tradicionalmente se cree que el calostro no ha sido bueno para el bebé. Había que expulsarlo en su totalidad antes de dar el pecho al recién nacido. Por lo tanto, se tenía la costumbre de no amamantar al neonato en los primeros días tras el parto (Sathiankoset 1996). Sin embargo, hoy en día se ha sabido que el calostro es mucho más saludable para el bebé que la leche materna de otros periodos. El periodo durante el cual se da el pecho al niño depende de cada madre, pero es común durante tres meses tras el parto. Después, debido a que la madre en la mayoría de los casos debe reanudar su vida laboral, el bebé será alimentado a base de biberón (Waikagul 2008).

Con respecto al entierro de la placenta, esta costumbre tiene como objetivo que el niño no se olvide de cuál es su origen. En la actualidad, esta práctica es cada vez menos frecuente porque el parto tiene lugar en el hospital y no en los domicilios con la ayuda de una comadrona. Se presta más atención en el cuidado para la madre y el recién nacido que en el entierro de la placenta como, por ejemplo, la nutrición y el cuidado de la salud de ambos. En la mayoría de los casos, la gente opta por no practicar esta costumbre, excepto en algunas zonas rurales como en el norte o el noreste del país (Marnpae 2011). Los lugares de entierro se determinan de acuerdo con la creencia de cada región del país, pudiéndose enterrar la placenta en ciertos lugares como por ejemplo, bajo una escalera de la casa (con propósito de que el niño tenga costumbre de permanecer en la casa o evitar el peligro si algún día se cayera por la escalera), bajo un árbol muy grande (para que el niño tenga una vida feliz y tan larga como el árbol), evitar un lugar bajo el árbol (con el fin de prevenir la subida al árbol del niño que luego puede acarrear un peligro), etc. (Sathiankoset 1996).

\subsection{Creencias tailandesas de pre-embarazo, embarazo, parto y posparto}

Chawanpaiboon (2010a) afirma que debido a la carencia de avances en la medicina en el pasado, los antepasados han creado creencias del embarazo. Son creencias que ellos aprendieron de sus experiencias, entornos y prácticas con el fin de evitar la inseguridad de sus descendientes y además para que las embarazadas cuidaran mucho su salud y la del niño. La misma autora, además, confirma que algunas creencias son útiles, algunas ilógicas y otras han sido modificadas o añadidas según la época del tiempo.

En lo que sigue trataremos las creencias existentes en la sociedad tailandesa sobre el embarazo en cuatro periodos: pre-embarazo, embarazo, parto y posparto, que hemos recopilado de varias fuentes (Chawanpaiboon 2010a, 2010b, Sathiankoset 1996).

\subsubsection{Pre-embarazo}

- Pedir ayuda a Buda o/y espíritus para que les ayuden a quedarse embarazadas.

- Pasar debajo del vientre de un elefante para tener hijos y que éstos gocen de muchos años de vida.

\subsubsection{Durante el embarazo}

Beber mucha cantidad de zumo de coco para que su bebé tenga la piel limpia y no tenga ninguna grasa blanca cuando nazca. Esto sirve para que el niño tenga buena piel. Lo cierto es que se quitará la grasa del bebé cuando cumpla el periodo de embarazo. Sin embargo, en caso de que se dé a luz con mucha antelación a los nueve meses, el bebé tendrá dicha grasa porque no le ha dado tiempo a quitársela aún. En la realidad, esto sólo es una creencia, pues científicamente se ha demostrado que el zumo de coco no aporta estas propiedades.

- Se prohíbe cometer actos considerados como malos, por ejemplo, matar a los animales, pescar, etc. 
- Está prohibida la asistencia de las embarazadas a la cremación o al funeral, ya que las mujeres podrían tener preocupación o estrés, y esto podría ocasionar una alteración en la salud del feto. Se puede encontrar esta creencia en casi todas las regiones del país. La diferencia es el motivo de la prohibición de la asistencia, por ejemplo, se teme que los espíritus ataquen y estén en el cuerpo de las madres o se teme que los espíritus malos del funeral sigan a las embarazadas. Sin embargo, si lo consideramos psicológicamente, los antepasados se preocupaban por la salud de la madre y del bebé porque sería muy probable que la madre tuviera estrés o se pusiera triste por culpa del ambiente entristecido del funeral y de la imagen desagradable plasmada ante sus ojos.

- En algunas provincias se ata en la muñeca de la madre un hilo sagrado (สายสิญจน์ [sa:jsin]) para proteger al niño de los peligros y los espíritus.

- No se toman comidas o sustancias que lleven el color negro si se desea tener a un niño con color de piel blanco ${ }^{1}$.

- Se debe suprimir la relación sexual durante el embarazo porque se cree que puede ocasionar el aborto o la minusvalía en el bebé. Lo cierto es que se pueden mantener relaciones sexuales, pero deben tenerse de forma suave y con una posición que no genere presión en el útero.

- Para saber el sexo del hijo, se pronostica por el carácter de ombligo (pues antes no había la tecnología de la ecografía). Si el ombligo está de forma convexa, sería un niño y en caso contrario, sería una niña. En la actualidad esta creencia sigue existiendo aunque no tiene nada que ver con la ciencia.

- Se considera que las medicinas con receta, que tienen la finalidad del cuidado durante el embarazo, ocasionarán el aumento de peso en las embarazadas. La verdad es que no es la creencia correcta. Ellas aumentan de peso debido al incremento de las hormonas durante el embarazo y por los hábitos que suelen tener las embarazadas, es decir, comer mucho y dormir fácilmente.

- En caso de las madres con un nivel cultural bajo, ellas consideran que el cuidado prenatal no es imprescindible si no ocurre ningún problema durante el embarazo. Algunas madres van a la consulta del médico para la visita prenatal en los últimos meses del embarazo e incluso algunas nunca han recibido dicho cuidado.

- Existe la preocupación del uso frecuente de la ecografía, ya que podría ocasionar problemas o minusvalía en el bebé. Lo cierto es que el aparato con el cual se realiza la ecografía no perjudica al bebé, pues no tiene rayos $\mathrm{X}$.

\subsubsection{Parto}

- En algunos casos, se considera que la cesárea es mejor que el parto vaginal, porque las parturientas tienen preocupación por la extensión de la vagina y no quieren hacer el parto de manera natural.

- Se cree que si se realiza la cesárea, el neonato será buena persona, inteligente y fuerte, sobre todo cuando se dé a luz a la hora auspiciosa según cálculos astrológicos. Actualmente cada vez es más popular realizar la cesárea a la hora auspiciosa según los cálculos astrológicos.

- Beber agua sagrada (el agua que ha sido recitada en el cántico por el monje budista) ayudará a no tener el dolor y a dar a luz con más facilidad.

- Se deben abrir todas las puertas, ventanas, armarios y cajones del domicilio. También cualquier persona debe evitar sentarse en la escalera del mismo a fin de facilitar el alumbramiento. Esta creencia está cada vez más extinguida.

\footnotetext{
${ }^{1}$ El canon de belleza actual en Tailandia es que cuánto más blanca sea la piel de una persona, más bella se considera. Es un pensamiento diferente al de los países occidentales, que en muchos casos consideran que tener la piel morena es un indicativo de belleza.
} 


\subsubsection{Posparto}

- Se evitan los cumplidos y alabanzas al neonato, utilizando esto como estrategia para evitar el secuestro de los espíritus. Se debería decir al bebé algo contrario a su belleza o cosas por el estilo como, por ejemplo, iqué feo este niño! en vez de iqué divino/ majo este niño!, aunque de hecho el bebé sea muy majo. Hoy en día existe todavía esta creencia en la sociedad tailandesa. Sin embargo, cuando se hace una visita a la madre y al recién nacido, debería preguntarse si aún se cree en el efecto adverso del elogio al niño y el secuestro de los espíritus, por si acaso no están satisfechos cuando escuchen algo contrario a lo que esperan.

- Es una creencia incorrecta el consumo de licor de hierba tailandés (ยาคอง [ya:-do:y]) o una medicina que sirve exclusivamente para expulsar los loquios después del parto. Se cree que los loquios son sustancias malas y hay que expulsarlos en gran cantidad. Hay muchas nuevas madres que han sido ingresadas en el hospital a causa de tener una hemorragia por haber bebido dicha medicina. El alcohol en el licor de hierba o la medicina puede pasar a la leche materna y provocar las alteraciones en el sueño de bebé, trastornos del desarrollo neurológico, etc.

- En caso de las parturientas de origen chino, deben mantenerse en sus domicilios un mes sin salir a ningún lado y evitar bañarse y lavarse el pelo durante un mes.

- Después del parto algunas madres optan por un cuidado postparto con los métodos de medicina tradicional, como la práctica llamada การอยู่ไฟ [ka:n-ju:-faj], esto es, que la madre esté tumbada junto al fuego y que ella tome baños de vapor con hierbas para cuidarse.

\section{Conclusiones}

La comunicación y la cultura tienen impacto sobre las consecuencias clínicas de la relación entre el médico y el paciente. Con el objeto de proporcionar una atención de la salud óptima a todos los clientes, cualquier persona que se involucre con la atención de la salud (personal sanitario, intérprete, mediador y trabajador social) debe tener en cuenta la diversidad cultural, sobre todo cuando los participantes de una interacción proceden de diferentes comunidades lingüísticas y/o culturales, ya que los usuarios traen consigo a la sociedad receptora sus estilos comunicativos, culturas sanitarias, conceptos de salud que a veces difieren significativamente de los correspondientes a los autóctonos. Poseer conocimientos culturales de los participantes en encuentros interculturales se considera uno de los pasos más significativos para lograr una comunicación intercultural eficaz y adecuada. Consecuentemente, se podría confirmar que es necesaria la incorporación de conocimientos culturales en el ámbito sanitario.

\section{Referencias}

Angelilli, C. V. 2004. Medical Interpreting and Cross-cultural Communication. Nueva York: Cambridge University Press.

Chawanpaiboon, S. 2010a. t6in-ru: kwa:m-t ${ }^{\mathrm{h}} u: \mathrm{k}^{\mathrm{h}} \mathrm{a}-\mathrm{na}$ toan-k ${ }^{\mathrm{h}}$ an (toon-thi:1) [¿Son de verdad las creencias durante embarazo? ( $1^{\mathrm{a}}$ parte)] Faculty of Medicine, Siriraj Hospital.

[Disponible en: http://www.si.mahidol.ac.th/sidoctor/e-pl/articledetail.asp?

$\underline{\mathrm{id}=760}$ ]

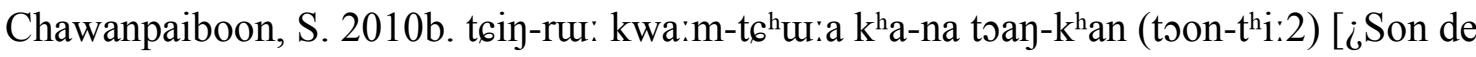


verdad las creencias durante embarazo? ( $2^{\mathrm{a}}$ parte)] Faculty of Medicine, Siriraj Hospital. [Disponible en: http://www.si.mahidol.ac.th/sidoctor/epl/articledetail.asp?id=761]

Chen, G. M., y W. J. Starosta. 1996. Intercultural communication competence: A Synthesis.

Communication Yearbook 19: 353-384.

Chen, G. M. y W. J. Starosta. 2005. Foundations of Intercultural Communication. Maryland: University Press of America.

Chokevivat, V. y A. Chuthaputti. 2005. The Role of Thai Traditional Medicine in Health Promotion. Department for the Development of Thai Traditional and Alternative Medicine, Ministry of Public Health, 6GCHP Bangkok Thailand: 7-11.Aug 2005. [Disponible en: http://www.thaihealingalliance.com/membersonly/Research_and_Other_Items_of_I nterest/The\%20Role\%20ofThai\%20Traditional\%20Medicine\%20in\%20Health\%20 Promotion, \%20by\%20Vichai\%20Chokevivat\%20and\%20Anchalee\%20Chuthaputti .pdf]

Flores, G. 2000. Cultural and the patient-physician relationship: achieving cultural competency in healthcare. Journal of Pediatrics 136: 14-23.

Grupo CRIT. 2006. Cultura cara a cara: Relatos y actividades para la comunicación intercultural. Madrid: Edinumen.

Gudykunst, W. B. 1998. Bridging differences: Effective intergroup communication. Thousand Oaks, CA: Sage.

Haffner, L. 1992. Translation Is Not Enough: Interpreting in a Medical Setting. Western Journal of Medicine 157: 225-230.

Hofstede, G. 1997 (1991). Cultures and organizations: Software of the mind. Nueva York: McGraw-Hill.

Lustig, M. W. y J. Koester. 2010. Intercultural Competence: Interpersonal Communication Across Cultures. Boston: Allyn \& Bacon.

Marnpae, M. 2011. Una aproximación descriptiva a la medicina tradicional tailandesa: aspectos culturales de interés para la traducción y la interpretación. Memoria del máster, Universidad de Alcalá.

Purnell, D. y B. J. Paulanka, eds. 1998. Transcultural Health Care: A Culturally Competent Approach. Filadelfia: F.A. Davis 1998).

Raga Gimeno, F. y D. Sales Salvador. 2010. El componente cultural en la formación para la interpretación y la mediación intercultural en el ámbito sanitario. En M. Carreras i Goicoechea y M. E. Pérez Vázquez (eds.), La mediación lingüística y cultural y su didáctica. Un nuevo reto para la Universidad. 165-192. Bolonia: Bononia University Press.

Salguero, C. P. 2003. A Thai Herbal. Escocia: Findhorn Press.

Salguero, C. P. 2006. The Spiritual Healing of Traditional Thailand. Escocia: Findhorn Press. Salguero, C. P. 2007. Traditional Thai Medicine. EE.UU: Hohm Press.

Samovar, L. A., R. E. Porter y E. R. McDaniel, eds. 2007: Communication between cultures. Belmont, CA: Thomson Wadsworth.

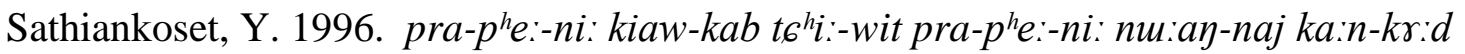

[Las costumbres de vida y las costumbres de nacimiento]. Bangkok: Siam Press.

Spencer-Oatey, H. y P. Franklin. 2009. Intercultural Interaction: A Multidisciplinary Approach to Intercultural Communication. Hampshire: Palgrave Macmillan.

Spitzberg, B. H. y W. R. Cupach. 1984. Interpersonal communication competence. Beverly Hills, CA: Sage.

Ting-Toomey, S. 1999. Communicating across Cultures. Nueva York: The Guilford Press. Triandis, H. C. 1977. Subjective culture and interpersonal relations across cultures. Annuals 
of the New York Academy of Sciences 285: 418-434.

Waikagul, J. 2008 (1994). Thailand (Kingdom of). En C. Erikson D'Avanzo (ed.), Pocket guide to Cultural Health Assessment. 708-712. St. Louis, Missouri: Mosby, Elsevier.

Wiseman, R. L. 2002. Intercultural Communication Competence. En W. B. Gudykunst y B.

Mody (eds.), Handbook of international and intercultural communication. 207-224.

Thousand Oaks/ Londres/ Nueva Delhi: Sage Publications. 